\title{
STATISTICAL INVESTIGATION OF BUBBLE COLLAPSE AND CAVITATION EROSION EFFECT
}

\section{Y. J. ZHANG}

China Ship Scientific Research Centre, Wuxi, Jiangsu (China)

S. C. LI*

North China Institute of Water Conservancy and Power, Handan, Hebei (China)

F. G. HAMMITT

Cavitation and Multiphase Flow Laboratory, Department of Mechanical Engineering and Applied Mechanics, University of Michigan, Ann Arbor, MI (U.S.A.)

(Received August 9, 1988; revised February 9, 1989; accepted March 21, 1989)

\section{Summary}

Including liquid compressibility, an approximate expression for acoustic energy radiated from a single collapsing bubble was derived based on the Noltingk-Neppiras approach and experimental results. The statistical distributions of pulsation amplitudes and acoustic energy emitted from multibubble collapses were analysed using the random pulse train model, and compared with new experimental results. These statistical considerations were used in analysis of the cavitation erosion effect.

\section{Introduction}

The shock wave radiated from collapsing bubbles is one of the main factors contributing to cavitation erosion. The dynamics of single bubbles at the collapse stage has been investigated experimentally by using electricspark and laser-induced methods reported in the literature [1 - 3]. However, as shown by the experimental results, the values of maximum pressure amplitudes, even emitted from those bubbles which had the same maximum radius, were still quite scattered. This is probably caused by the effects of many other uncertain factors such as the air content in the bubble, the stability of the spherical shape of bubbles during collapse etc.

It is well known that the cavitation phenomenon in flow systems is much more complicated than in single-bubble generators. To estimate this highly stochastic acoustic behaviour for the multibubble collapsing process, a random pulse train model was set up several years ago $[4,5]$. It is useful for the mathematical treatment of such a random process. For a better

*Visiting Fellow, Department of Engineering, University of Warwick, U.K. 
understanding of these stochastic features, a statistical study of multibubble collapse and the cavitation erosion effect, based on the pulse train model, was thus initiated.

\section{Single bubble collapse}

When a single bubble collapses, a considerable portion of the potential energy stored in the bubble is transformed into acoustic energy, radiating strong acoustic pulsations into the flow field. To evaluate the radiated acoustic energy, Rayleigh's classical theory on bubble dynamics may be used to calculate the volumetric acceleration and radial velocity of the bubble, and the Noltingk-Neppiras equation to estimate the minimum radius at collapse [6]. Thus the acoustic energy $E_{\mathrm{ac}}$ can be expressed as

$\frac{E_{\mathrm{ac}}}{E_{\mathrm{pot}}}=\frac{1}{c}\left(\frac{P_{\mathrm{h}}}{\rho}\right)^{1 / 2} \mathrm{~F}\left(x_{\min }\right)$

where $E_{\mathrm{pot}}$ is the potential energy of the collapsing bubble. Then

$E_{\text {pot }}=\frac{4}{3} \pi a_{\max }{ }^{3} P_{\mathrm{h}}$

and $x$ can be defined as the non-dimensional volume

$x=\left(\frac{a}{a_{\max }}\right)^{3}$

The function $F\left(x_{\min }\right)$, from classical theory [6], is

$\mathrm{F}\left(x_{\min }\right)=-\frac{1}{6} \frac{2}{3} \int_{1}^{x_{\min }} \frac{1-8 x+16 x^{2}}{x\{x(1-x)\}^{1 / 2}} \mathrm{~d} x$

Following the Noltingk-Neppiras method, $a_{\min }$ can be approximated as

$\frac{a_{\min }}{a_{\max }}=\left(\frac{Q}{(\gamma-1) P_{\mathrm{h}}}\right)^{1 / 3(\gamma-1)}\left(1+\frac{Q}{(\gamma-1) P_{\mathrm{h}}}\right)^{-1 / 3(\gamma-1)}$

If the partial pressure of gas in the bubble $Q$ is much less than the local pressure in the flow field, i.e. $Q \ll P_{\mathrm{h}}$, and letting the adiabatic index $\gamma \approx 4 / 3$, eqns. (4) and (5) can be simplified to

$\frac{a_{\min }}{a_{\max }} \approx 3 \frac{Q}{P_{\mathrm{b}}}$

$\mathrm{F}\left(x_{\min }\right)=\frac{1}{3}\left(\frac{2}{3}\right)^{1 / 2} x_{\min }{ }^{-1 / 2}$

The following relation is then deduced:

$\frac{E_{\mathrm{ac}}}{(4 / 3) \pi P_{\mathrm{h}} a_{\max }{ }^{3}}=\frac{1}{27 c}\left(\frac{2 P_{\mathrm{h}}}{\rho}\right)^{1 / 2}\left(\frac{P_{\mathrm{h}}}{Q}\right)^{3 / 2}$ 
As the cavitation bubble is developed initially from a gas nucleus with radius $a_{\mathrm{e}}$, at the equilibrium pressure $P_{\mathrm{e}}$ it is approximately true, neglecting surface tension, that

$Q=P_{\mathrm{e}}\left(\frac{a_{\mathrm{e}}}{a_{\max }}\right)^{3}$

The Noltingk-Neppiras approach assumes an incompressible fluid. However, at the final stage of collapse, the compressibility of the surrounding fluid will significantly depress the high speed contracting motion of the bubble. Thus eqn. (8) gives too high an evaluation for the radiated acoustic energy. This is recognized in a past numerical analysis including compressibility [7]. As an alternative, we let eqn. (8) have the following form:

$\frac{E_{\mathrm{ac}}}{(4 / 3) \pi P_{\mathrm{h}} a_{\max }{ }^{3}}=\frac{1}{27}\left(\frac{2 P_{\mathrm{h}}}{\rho c^{2}}\right)^{1 / 2}\left(\frac{P_{\mathrm{h}}}{Q}\right)^{\beta}$

Here $\beta$ is an empirical index, $0<\beta<3 / 2$, to include the effect of liquid compressibility. As shown by many experimental results, the acoustic pulsations emitted from bubble collapse decay rapidly. For example, it was reported [3] that the ratio of the bubble energy of a subsequent pulsation to the previous, $E_{\mathrm{ac}}{ }^{(2)} / E_{\mathrm{ac}}{ }^{(1)}=0.01-0.05$, in the case of spark-generated bubbles, and $E_{\mathrm{ac}}{ }^{(2)} / E_{\mathrm{ac}}{ }^{(1)}=0.01-0.07$, for laser-generated bubbles. Thus, from the viewpoint of cavitation damage, it is reasonable to consider only the first pulsation. The first pulsation can then be written in the following form [2]:

$p(t)= \begin{cases}p_{\max } \mathrm{e}^{-t / \alpha} & t \geqslant 0 \\ 0 & t<0\end{cases}$

where $\alpha$ can be approximately expressed as

$\alpha=k a_{\max }\left(\frac{\rho}{P_{\mathbf{h}}}\right)^{1 / 2}$

Thus

$E_{\mathrm{ac}}=\frac{4 \pi r^{2}}{\rho c} \int_{0}^{\infty} p^{2}(t) \mathrm{d} t=\frac{2 \pi r^{2}}{\rho c} \alpha p_{\max }{ }^{2}$

The acoustic peak value $p_{\mathrm{m}}$ is approximately proportional to $a_{\max }{ }^{3 / 2}$. From the experimental observations [1 - 3], it has the form

$p_{\max }=K a_{\max }{ }^{3 / 2}$

Comparing eqns. (10) and (13), and considering eqns. (9) and (14), we have

$\beta=\frac{1}{3}$

$K=\frac{2^{3 / 4} P_{\mathrm{h}}{ }^{7 / 6}}{9 r k^{1 / 2} P_{\mathrm{e}}^{1 / 6} a_{\mathrm{e}}^{1 / 2}}$ 
3. Pulsation trains at collapse

The distribution of gas nuclei may be expressed as [8]

$W_{\mathrm{a}}(X)= \begin{cases}B X^{-\nu} & a_{\mathrm{e} 1} \leqslant X \leqslant a_{\mathrm{e} 2} \\ 0 & X<a_{\mathrm{e} 1} \quad \text { or } \quad X>a_{\mathrm{e} 2}\end{cases}$

where

$B=(-\nu+1)\left(a_{\mathrm{e} 2}{ }^{-\nu+1}-a_{\mathrm{e} 1}{ }^{-v+1}\right)^{-1}$

The critical size $a^{*}$ for unstable growth can also be computed [9].

If the density of gas nuclei is $N$, then the effective density of nuclei contributing to cavitation $N_{\text {eff }}$ can be calculated

$N_{\mathrm{eff}}=N \int_{a^{*}}^{a_{\mathrm{e} 2}} B X^{-\nu} \mathrm{d} X=\frac{a_{\mathrm{e} 2}^{-\nu+1}-\left(a^{*}\right)^{-\nu+1}}{a_{\mathrm{e} 2}^{-\nu+1}-a_{\mathrm{e} 1}{ }^{-\nu+1}} N$

In a constant and uniform low pressure field with pressure $P_{1}$, the bubble finally reaches its maximum size $a_{\max }$ [6]

$a_{\max } \approx\left(\frac{2}{3} \frac{P_{1}}{\rho}\right)^{1 / 2} T$

The pressure pulses radiated from collapsing bubbles in a high pressure field $P_{\mathrm{h}}$ are treated as a train of pulsations with the exponential decay form defined in eqn. (11), but with random peak values $p_{m}$ and random time intervals $\Delta T$ between successive pulses.

For randomly distributed bubbles, the average distance between two neighbouring bubbles is given by

$d^{-1}=N_{\text {eff }}^{1 / 3}$

Thus the average time interval $\overline{\Delta T}$ is

$\overline{\Delta T}=\frac{d}{V}$

Now we estimate the amplitude probability density distribution of multipulses, i.e. the pulse train, under the assumption of a stationary random process. A cut-off value of the amplitude $p_{\mathrm{c}}$ is introduced (Fig. 1). We are now only interested in the distribution of those amplitudes higher than $p_{c}$, since those below $p_{\mathrm{c}}$ scarcely contribute to cavitation damage, but rather to noise. With this consideration, the amplitude density distribution $W_{\mathrm{I}}(X)$ may be expressed as

$W_{\mathrm{I}}(X)=\mu \frac{\alpha}{X} \int_{X}^{\infty} W_{\mathrm{p}}(X) \mathrm{d} X \quad\left(X>p_{\mathrm{c}}\right)$

where $W_{p}(X)$ is the density distribution of pulse peaks and $\mu$ the normalization factor. It may be found from eqns. (14) and (17) that 
P

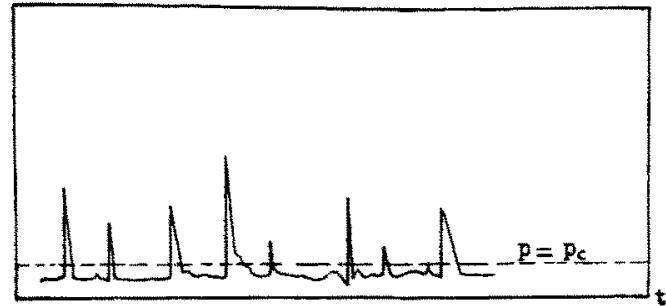

Fig. 1. Cavitation pulse train.

$$
\begin{aligned}
& W_{\mathrm{p}}(X)=W_{\mathrm{a}}\left\{p_{\mathrm{m}}^{-1}(X)\right\}\left|\frac{\mathrm{d} p_{\mathrm{m}}^{-1}(X)}{\mathrm{d} X}\right| \\
& = \begin{cases}2 B_{1} K^{2(1-\nu)} X^{2 \nu-3} & \frac{K}{a_{\mathrm{e} 2}^{1 / 2}} \leqslant X \leqslant \frac{K}{\left(a^{*}\right)^{1 / 2}} \\
0 & X<\frac{K}{a_{\mathrm{e} 2}^{1 / 2}} \quad \text { or } \quad X>\frac{K}{\left(a^{*}\right)^{1 / 2}}\end{cases}
\end{aligned}
$$

$B_{1}=(-\nu+1)\left\{a_{\mathrm{e} 2}{ }^{-\nu+1}-\left(a^{*}\right)^{-\nu+1}\right\}^{-1}$

$\mu^{-1}=\int_{0}^{\infty} \alpha W_{\mathrm{p}}(X) \ln \frac{X}{X_{\mathrm{o}}} \mathrm{d} X$

Substituting eqn. (25) into eqns. (23) and (24), it is found that

$$
W_{\mathrm{I}}(X)= \begin{cases}\frac{\mu}{X} & p_{\mathrm{c}}<X<\frac{K}{a_{\mathrm{e} 2}{ }^{1 / 2}} \\ \frac{\mu B_{1}}{(\nu-1) X}\left\{\left(a^{*}\right)^{1-\nu}-\left(\frac{X}{K}\right)^{2(\nu-1)}\right\} & \frac{K}{a_{\mathrm{e} 2}^{1 / 2}} \leqslant X \leqslant \frac{K}{\left(a^{*}\right)^{1 / 2}} \\ 0 & X>\frac{K}{\left(a^{*}\right)^{1 / 2}}\end{cases}
$$

where

$\mu=\left[\frac{B_{1}}{\nu-1}\left\{\left(a^{*}\right)^{1-\nu} \ln \frac{K}{\left(a^{*}\right)^{1 / 2}}-a_{\mathrm{e} 2}^{1-\nu} \ln \frac{K}{a_{\mathrm{e} 2}{ }^{1 / 2}}\right\}-\frac{1}{2(\nu-1)}-\ln p_{\mathrm{c}}\right]^{-1}$

It is convenient to represent eqn. (27) in a dimensionless form. Let

$$
X=\bar{X} X_{0} \quad p_{\mathrm{c}}=\bar{p}_{\mathrm{c}} X_{0} \quad \text { and } \quad \bar{p}^{*}=\left(\frac{a^{*}}{a_{\mathrm{e} 2}}\right)^{1 / 2}
$$




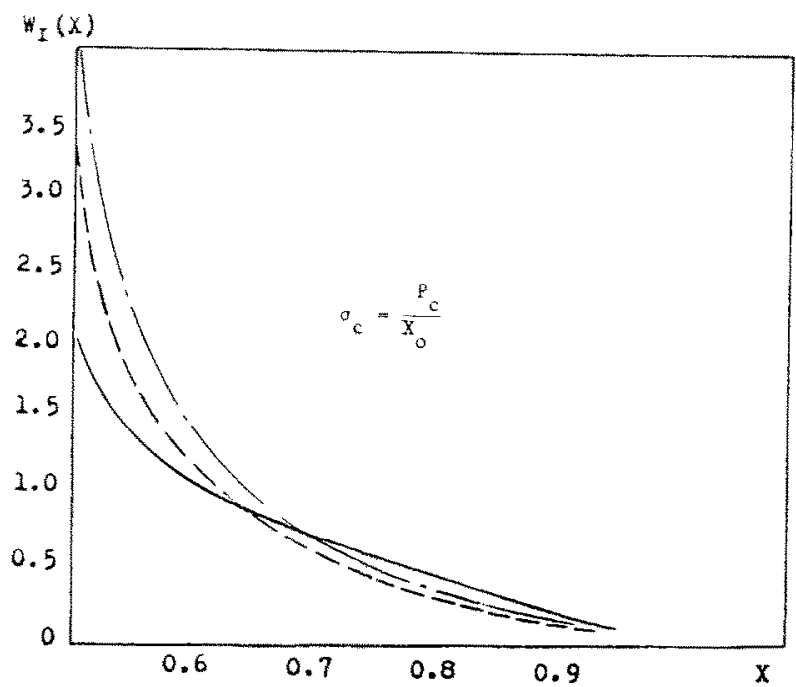

Fig. 2. Dimensionless amplitude distribution: $-\rightarrow$, theory; $-\cdots$, experimental results (ref. 10 ) $, \sigma_{c}=0.030, X_{0}=26.85$ bar; - - , experimental results (ref. 10), $\sigma_{c}=0.025$, $x_{0}=28.35$ bar.

Then eqn. (27) becomes

$W_{\mathrm{I}}(\bar{X})= \begin{cases}\frac{C}{\bar{X}} & \bar{p}_{\mathrm{c}}<\bar{X}<\overline{p^{*}} \\ \frac{C}{\bar{X}} \frac{1-(\bar{X})^{2(\nu-1)}}{1-\left(\bar{p}^{*}\right)^{2(\nu-1)}} & \overline{p^{*}} \leqslant \bar{X} \leqslant 1 \\ 0 & \bar{X}>1\end{cases}$

where

$C=-\frac{1}{X_{0}}\left\{\frac{1}{2(\nu-1)}+\ln \bar{p}_{\mathrm{c}}+\frac{\left(\overline{p^{*}}\right)^{2(\nu-1)}}{1-\left(\overline{p^{*}}\right)^{2(\nu-1)}} \ln \bar{p}^{*}\right\}$

Comparison of these theoretical results with the measured pulse height spectra reported in ref. 10 shows (Fig. 2) that for the high amplitudes, the experimental curve has a power function form similar to the theoretical curve of eqn. (29). However, for the low amplitudes, the measured curve approximates an exponential rather than a power form. This discrepancy is presumably caused by the peak detection method used in ref. 10. It is an even-time-interval sampling method. Therefore it is not very precise for small peak counting.

\section{Cavitation erosion}

It was reported [10] that cavitation damage was linearly proportional to cavitation acoustic power calculated from pulse height spectra. Thus the 
cavitation erosion efficiency $\eta_{c}$ is introduced [11 - 13] to characterize the ratio of erosion to acoustic power, or the ratio of the portion of energy absorbed by the material to the pressure pulse energy adjacent to damaged material. If we assume that $\eta_{\mathrm{c}}$ depends only on the properties of the material, and is basically independent of the detailed flow conditions, then it should be possible to use the acoustical method to predict cavitation erosion for an otherwise untested condition. Thus the stochastic characteristics of the cavitation acoustic power concerning the erosion can be statistically considered as follows.

From eqns. (13) and (24), the statistical distribution of acoustic energy emitted from collapsing bubbles can be reduced as

$$
W_{E}(X)= \begin{cases}B_{1} K_{1}^{2(1-\nu)} X^{\nu-2} & \frac{K_{1}{ }^{2}}{a_{\mathrm{e} 2}} \leqslant X \leqslant \frac{K_{1}{ }^{2}}{a^{*}} \\ 0 & X<\frac{K_{1}{ }^{2}}{a_{\mathrm{e} 2}} \quad \text { or } \quad X>\frac{K_{1}{ }^{2}}{a^{*}}\end{cases}
$$

where

$K_{1}=\left(\frac{4 \pi \alpha}{\rho c}\right)^{1 / 2} K$

For a given acoustic energy distribution and any chosen time interval, the total acoustic energy is proportional to the number of collapsing bubbles. Therefore the damage to the material depends on the total number of collapsing bubbles $M$ which is also a stochastic variable. It is known [5] that the distribution of time intervals between pairs of successive bubblecollapsing events obeys an exponential law with the mean value as given in eqn. (22). Therefore the distribution of the time required for completing a number $M$ of collapsing bubbles $W_{M}(X)$ should have a gamma distribution form, i.e.

$$
W_{M}(X)=\frac{\lambda^{M}}{\Gamma(M)} X^{M-1} \mathrm{e}^{-\lambda X} \quad(X>0)
$$

where $\lambda^{-1}=\overline{\Delta T}$, defined in eqn. (22). Equation (33) shows that when the value of $M$ increases, $W_{M}(X)$ spreads more widely with a smaller peak at $X=(M-1) / \lambda \approx M \overline{\Delta T}$. This coincides quite well with the experimental observation that cavitation damage test results, if not treated statistically, are always quite scattered, even under carefully controlled test conditions, especially those referring to long time periods. Thus the total energy $\epsilon$ required for certain damage in terms of the number of collapsing bubbles $M$ is

$$
\epsilon=M \int_{0}^{\infty} X W_{E}(X) \mathrm{d} X=M \frac{\nu-1}{\nu} \frac{K_{1}^{2}}{a^{*}} \frac{1-\left(\overline{x^{*}}\right)^{2 \nu}}{1-\left(\overline{x^{*}}\right)^{2(\nu-1)}}
$$


We now estimate the influence of flow velocity $V$ on the total acoustic energy $\epsilon$. For the given testing period $\tau$ the average value of $M$ is equal to $\tau / \overline{\Delta T}$. Inserting this value into eqn. (34), we have

$\epsilon \propto \frac{P_{\mathbf{h}}{ }^{11 / 6} a_{\max }{ }^{4}}{\overline{\Delta T}}$

From eqns. (22) and (20), it follows that

$\overline{\Delta T} \propto V^{-1}$

$a_{\max } \propto V^{-1}$

For a turbo-impeller driven system, it is approximately true that

$P_{\mathrm{h}} \propto V^{2}$

Then we have

$\epsilon \propto V^{2 / 3}$

Comparing this with the experimental results from the University of Michigan venturi [10] where the relation $\epsilon \infty V^{0.2}$ was found, it now appears that the theoretical model should include details of the flow conditions, such as those in the actual flow device (venturi) to get more precise results.

\section{Conclusions}

(1) If we consider the compressibility of the liquid during bubble collapse, the acoustic energy emitted from a single collapsing bubble can be estimated from the relation in eqn. (10), in which $0<\beta<3 / 2$. For cavitation damage, the value of $\beta$ can be considered as $1 / 3$.

(2) For multibubble collapses, the statistical distribution of pulsation amplitudes has a power function form as shown in eqn. (29). It coincides well with experimental results for large amplitudes.

(3) The statistical distribution of acoustic energy emitted from multibubble collapses was derived using the random pulse train model, which has the function form shown in egn. (31).

(4) Cavitation damage to any particular material is proportional to the number of collapsing bubbles $M$. For a given time period, $M$ is a stochastic variable. However for a given value of $M$, the time required to achieve $M$ is also a stochastic variable with the statistical distribution of a gamma function (eqn. (33)). This is a good explanation of the fact that experimental results for cavitation damage are usually quite scattered.

(5) Cavitation damage to various materials could be predicted by estimating the acoustic energy based on the measurement of cavitation pulse spectra in the flow field to which the material is exposed. 


\section{References}

$1 \mathrm{M}$. Harrison, An experimental study of single bubble cavitation noise, d. Acoust. Soc. Am., 24 (6) (1952) $776 \cdot 782$.

2 R. H. Mellen, An experimental study of the collapse of a spherical cavity in water, J. Acoust. Soc. Am., 28 (3) (1956) 447 - 454.

3 V. S. Teslenko, Experimental Investigation of Bubble Collapse at Laser-induced Breakdown in Liquids, Cavitation and Inhomogeneities in Underwater Acoustics, Springer, Berlin, 1980.

4 V. I. I'ichev and V. P. Lesunovskii, On the noise spectra associated with hydrodynamic cavitation, Sou. Phys,-Acoust, 14 (1968) 25 - 28.

5 V. P. Morozov, Cavitation noise as a train of sound pulses generated at random times, Sov. Phys.-Acoust., 14 (1968) 361 - 365.

6 D. Ross, Mechanics of Underwater Noise, Pergamon, New York, 1976.

7 R. D. Ivany and F. G. Hammitt, Cavitation bubble collapse in viscous compressible liquids, numerical analysis, Trans. ASME, J. Basic Eng., Ser, D, 87 (1965) 977 - 985.

$8 \mathrm{~S}$. S. Pan and Y. J. Zhang, Similarity law for nuclei population in bubble cavitation inception, Int. Symp. on Cavitation in Hydraulic Structures and Turbomachinery, ASME, 1985.

9 M. S. Plesset, The dynamics of cavitation bubbles, J. Appl. Mech., Trans. ASME, 16 (1949) $228-231$.

10 M. K. De, Acoustic waves from hydrodynamic cavitation, Ph.D. Thesis, Nuclear Engineering Department, University of Michigan, 1980.

11 F. G. Hammitt, Cavitation and Multiphase Flow Phenomena, McGraw-Hill, New York, 1980 ,

12 F. G. Hammitt, Handbook of Fluids and Fluid Machinery, Chapter 8, Cavitation, Wiley, New York, 1983.

13 M. K. De and F. G. Hammitt, A new method for monitoring and correlating cavitation noise to erosion capability, Trans. ASME, J. Fluids Eng., 104 (4)(1982) 434-439.

\section{Appendix A: Nomenclature}

$\begin{array}{ll}a & \text { bubble radius } \\ c & \text { speed of sound } \\ E_{\mathrm{ac}} & \text { acoustic energy } \\ E_{\mathrm{pot}} & \text { potential energy } \\ N & \text { number density of gas nuclei } \\ N_{\mathrm{eff}} & \text { effective number density of gas nuclei } \\ p & \text { acoustic pressure } \\ P & \text { pressure of flow field } \\ Q & \text { partial pressure of gas in the bubble for radius, } a_{\max } \\ r & \text { space distance } \\ T & \text { bubble growth time } \\ \Delta T & \text { time interval between successive pulses } \\ V & \text { stream velocity } \\ x & \text { non-dimensional volume of bubble } \\ X & \text { random variable } \\ \text { Greek } & \text { symbols } \\ \alpha & \text { characteristic time of bubble collapse } \\ \gamma & \text { adiabatic index } \\ \rho & \text { mass density of water }\end{array}$

